\title{
Transmission Electron Microscope Study of Cold-Rolled Hadfield Steels*
}

\author{
By Zenji Nishiyama**, Muneo Oka** and Hiroshi Nakagawa***
}

\begin{abstract}
The structure of the Hadfield steel $(1.17 \% \mathrm{C}, 12.48 \% \mathrm{Mn})$ subjected to $30 \%$ cold-rolling at room temperature has been studied by means of transmission electron microscopy. In the electron micrograph a number of parallel fine bands $(50 \sim 100$

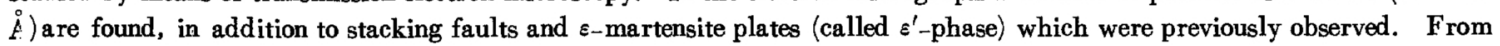
the result of the analysis by selected-area electron diffraction, the fine bands newly found are interpreted as deformation twins formed on the (111) planes of the matrix. The formation of such fine deformation twins is considered to cause work hardening of the Hadfield steel together with the formation of the $\varepsilon$-phase and the stacking faults.

(Received November 20, 1964)
\end{abstract}

\section{Introduction}

It is well known that the Hadfield steel can be so much hardened by cold-working that the steel is used as wear-resisting material. Much work ${ }^{(1) \sim(7)}$ has been made to make clear the mechanism of work hardening, but some questions still remain unsolved.

Colette ${ }^{(4)}$ et al., using $\mathrm{X}$-ray diffraction, detected $\alpha$ martensite (called $\alpha^{\prime}$-phase) and $\varepsilon$-martensite ( $\varepsilon^{\prime}$-phase) in the Hadfield steel subjected to more than $10 \%$ elongation at room temperature, and ascribed the work-hardening to the existence of these two phases.

Nishiyama and Shimizu ${ }^{(5)}$, as predicted in previous papers ${ }^{(3)}$, found stacking faults and thin $\varepsilon^{\prime}$-phase plates in a hammered manganese steel containing $0.97 \% \mathrm{C}$ and $9.75 \% \mathrm{Mn}$ by means of transmission electron microscopy, and suggested that their existence would play a part in the work-hardening. Using the same method, Roberts ${ }^{(7)}$ obtained the result that in a manganese steel containing $1.10 \% \mathrm{C}$ and $11.8 \% \mathrm{Mn}$ the $\alpha^{\prime}$-phase and stacking faults were produced by tension and the $\varepsilon^{\prime}$-phase by hammering. On the contrary, White and Honeycombe ${ }^{(6)}$ reported that they could not find such transformation products in various kinds of manganese steel deformed at room temperature or at $-196^{\circ} \mathrm{C}$, by means of the X-ray diffraction and the electron microscopic observation using the replica technique, and drew a conclusion that the work-hardening was due to the existence of stacking

* This paper has already been published in J.Japan Inst. Metals (in Japanese), 28(1964), 403.

** Institute of Scientific and Industrial Research, Osaka University, Sakai-shi, Osaka-fu, Japan.

*** Graduate Course of Engineering, Osaka University, Osaka, Japan.

(1) R. K. Buhr, S. L. Gertsmann and J. Reekie: Trans. ASM, 49 (1957), 706.

(2) H. M. Otte : Acta Met., 5(1957), 614.

(3) Z. Nishiyama, K. Shimizu, M. Oka and T. Hiromoto: J. Japan Inst. Metals, 22 (1958), 532; 23 (1959), 135 ; Mem. Inst. Sci. \& Ind. Res., Osaka Univ., 16 (1959), 73.

(4) G. Colette, C. Crussard, A. Kohn, J. Plateau, G. Pomey and M. Weisz : Rev. de Mét., 54 (1957), 6, 433.1

(5) Z. Nishiyama and K. Shimizu: J. Phys. Soc. Japan, 15 (1960), 1963.

(6) C. H. White and R. W. K. Honeycombe : J. Iron Steel Inst., $200(1962), 457$.

(7) W. N. Roberts : 5 th Intern. Conf. Electron Microscopy, (Philad.) (1962), HH-7.

Trans. J I M faults, the locking of dislocations by carbon atoms, and the interaction of stacking faults with these atoms.

As described above, there are dissenting views on the origin of the work hardening of manganese steel. To make clear this point, the present authors made a supplemental experiment using commercial manganese steels of Hadfield compositions. The result showed that deformation twins were produced by cold working, in addition to the stacking faults and the $\varepsilon^{\prime}$-phase which were previously observed in high manganese steel.

\section{Specimens and Experimental Method}

Specimens were prepared from an arc-melted cast steel containing $1.19 \% \mathrm{C}$ and $12.41 \% \mathrm{Mn}$ by the following procedures. To break the cast structure, the specimens were annealed in vacuum for $5 \mathrm{hr}$ at $1050^{\circ} \mathrm{C}$ after hotforging at $900^{\circ} \mathrm{C}$ at the forging ratio of 2 . They were again hot-forged at $800^{\circ} \sim 900^{\circ} \mathrm{C}$ to plates $1.5 \mathrm{~mm}$ thick, ground to the thickness of $1 \mathrm{~mm}$ to remove the decarburized surface layer, heated in vacuum for $4 \mathrm{hr}$ at 1050 ${ }^{\circ} \mathrm{C}$, quenched in water, and lastly cold-rolled to the thickness of $0.5 \mathrm{~mm}$. In order to make them austenitic, they were again heated at $1050^{\circ} \mathrm{C}$ for $1 \mathrm{hr}$, and quenched in water.

Deformation of the specimens accorded the cold-rolling method. The specimens quenched as above were coldrolled to the thickness of $0.35 \mathrm{~mm}$ corresponding to the $30 \%$ reduction. Finally, a chemical analysis was made to examine the non-decarburization during heat treatments. From the result that the specimens contained $1.17 \% \mathrm{C}$ and $12.48 \% \mathrm{Mn}$, it is evident that no appreciable decarburization did not take place.

Foils for electron microscopy were prepared electrolytically by Bollmann's method, using an electrolyte containing $200 \mathrm{cc}$ phosphoric acid and 100 gr chromic acid, at a current density $0.5 \mathrm{~A} / \mathrm{cm}^{2}$ and a voltage of $15 \mathrm{~V}$ at $90^{\circ} \mathrm{C}$. The electron microscope used was of the HU-11 type operated at $100 \mathrm{kV}$. For reference, the hardness was measured with Akashi Microvickers under a 200 gr load.

III. Experimental Results 


\section{Hardness}

The hardness of the specimens was $200 \sim 240 \mathrm{VHN}$ in the quenched austenitic state and $440 \sim 620 \mathrm{VHN}$ in the cold-rolled state. The optical micrograph of the latter showed a number of linear markings.

\section{Trınsmission electron microscope struct ures} and electron diffraction patterns

Photo. 1 (a) is a transmission electron micrograph of

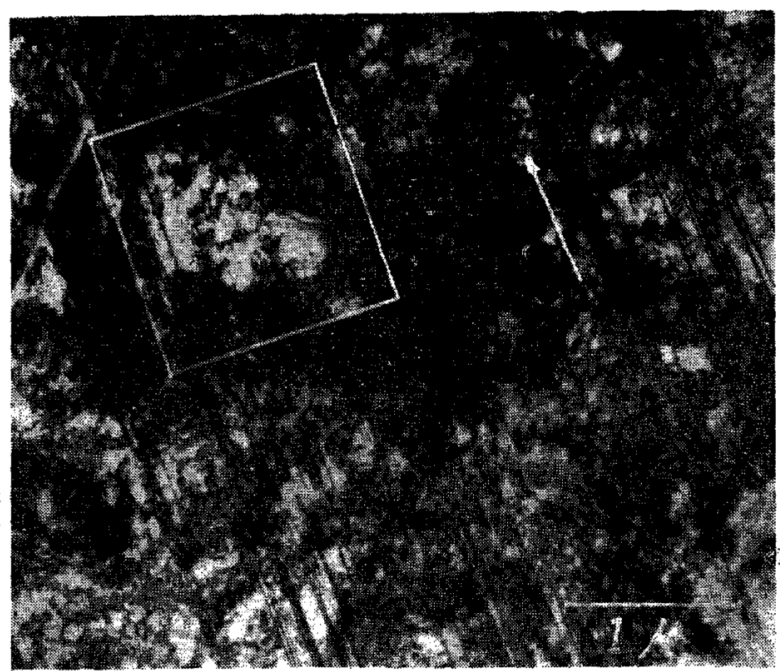

Photo. 1 (a) Transmission electron micrograph of Hadfield manganese steel subjected to $30 \%$ cold-rolling at room temperature, showing a parallel-line structure of deformation twins.

a cold-rolled specimen in which a number of fine bands parallel to the arrow $(\nwarrow)$ can be seen. These bands look rather sharp as compared with those previously report$\mathrm{ed}^{(5)}$ as $\varepsilon^{\prime}$-phase plates. An electron diffraction pattern of the white framed-area is given in Photo. 1 (b).

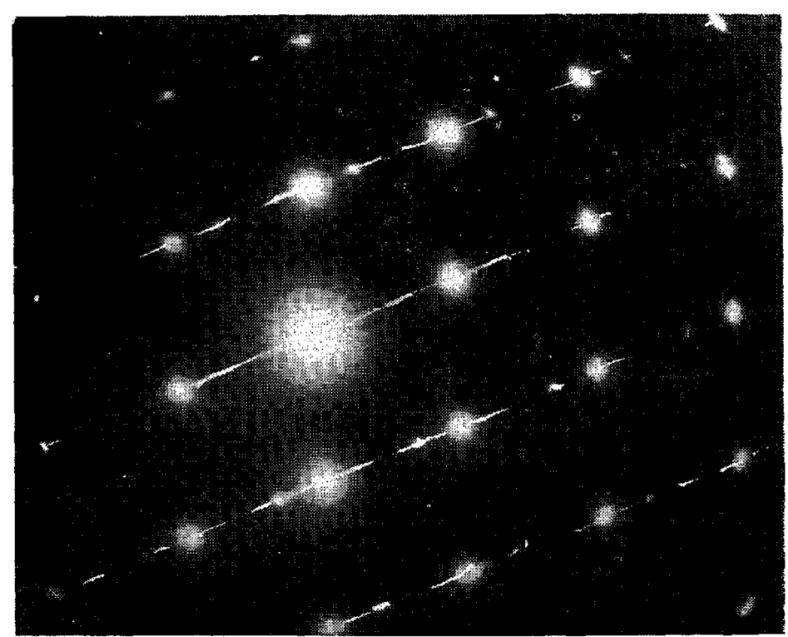

Photo .1.(b) Selected-area electron diffraction pattern of the framed area in Photo. 1(a).

As illustrated in Fig. 1, intense spots in this pattern are considered as reflections from the matrix, which shows the foil plane to be the $(1 \overline{1} 0)$ plane. The other spots weak in intensity are symmetrical in arrangement with the spots of the matrix about the trace of the (111) plane perpendicular to the foil plane. From the analysis of these patterns it is concluded that the fine bands observed in Photo. 1 (a) are deformation twins.
The thickness of these twins ranges between 50 and $100 \AA$ and their spacing lies between 100 and $200 \AA$ at the dense portion and between 300 and $400 \AA$ on the average. Since the diffraction spots are accompanied with streaks running perpendicular to the fine bands in (a), it seems that the twins are very thin or contain many faults parallel to the twinning plane.

Photo. 2 (a) is another electron micrograph in which broad bands are observed, overlapping each other in some

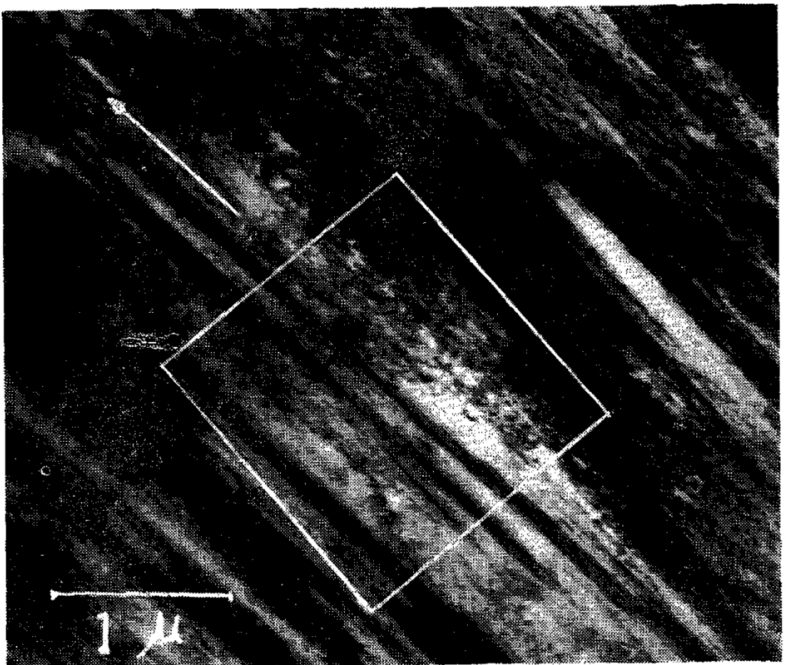

Photo. 2 (a) Transmission electron micrograph of Hadfield manganese steel subjected to $30 \%$ cold-rolling at room temperature, showing a parallel-line structure of deformation twin and $\varepsilon^{\prime}$-phase.

portions. The diffraction pattern, Photo. $2(b) *$, is very complicated, but the austenite spots can easily be selected. These spots show that the foil plane is the (101) plane and the direction of bands (marked by an arrow) is [101] and parallel to the trace of the (111) plane. It is, however, noted that all the bands are not exactly parallel but some

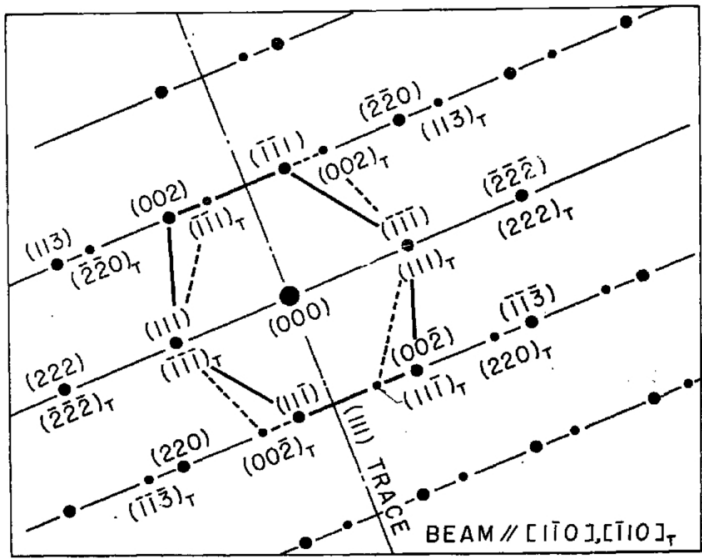

Fig. 1 Key diagram of Photo. 1(b). Suffix ' $T$ ' means the twin spot.

deviate slightly. This shows a high degree of working for the specimen. Therefore it was assumed for the indexing of diffraction spots that crystal parts in the foil were rotated. The diffraction pattern in Photo. 2 (b) can be interpreted from its key diagram, Fig. 2, in which

* Photo. 2 (b) was taken when the foil plane was inclined clockwise by $2^{\circ}$ about the $[\overline{101}]$ ( $\backslash$ ) from the orientation of Photo. $2(a)$. 
spots connected by full lines belong to the [101] zone of the matrix. If hexagonal close packed crystals whose basal planes are parallel to the (111) plane of the matrix (with Shoji-Nishiyama orientation relationships (111) // $\left.(0001)_{\varepsilon},[\overline{1} 2 \overline{1}] / /[10 \overline{1} 0]_{\varepsilon}\right)$ are contained in the speci-

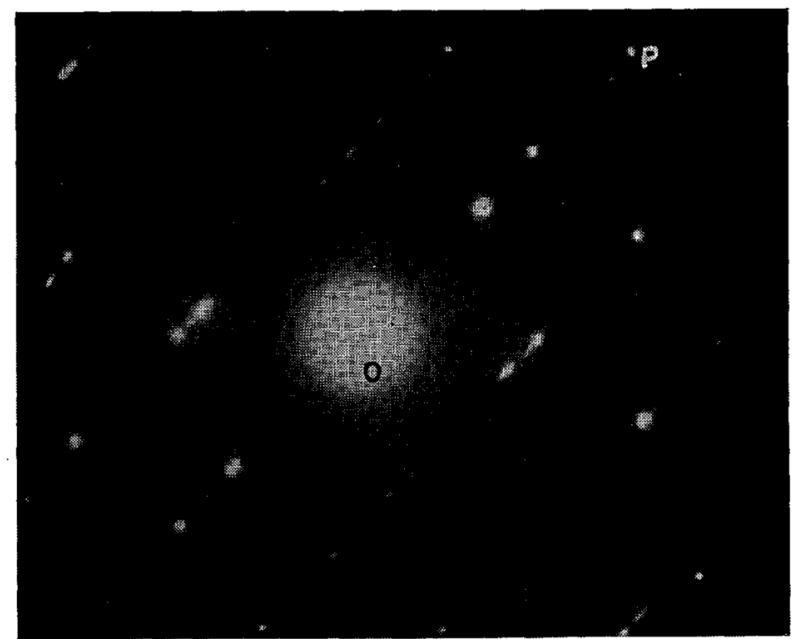

Photo. 2 (b) Selected-area electron diffraction pattern of the framed area in Photo. 2 (a).

men, their $(10 \overline{1} 2)_{\varepsilon}$ spot is expected to appear when the specimen is inclined by $9^{\circ} 55^{\prime}$ clockwise** about the ['101] axis. Actually it is observed in Photo. 2 (b). The zone axis of the hexagonal pattern to be observed is [10 $\overline{1} \overline{1}] \varepsilon$. The above consideration is also confirmed by the appearance of spots $(01 \overline{1} 1)_{\varepsilon}$ and $(\overline{1} 10 \overline{1})_{\varepsilon}$ which are connected by dot-dashed lines. Moreover, if deformation twins are present, spots belonging to the [100] zone will appear when the specimen is further inclined clockwise. Actually they are found, as shown by the spots connectied with dotted lines***. If the reflection spots from the $\varepsilon^{\prime}-$ phase or the twins for some reason, do not have definite positions but were elongated along the [111] direction in the reciprocal space it would not be possible to determine whether any spots other than those of the matrix arise from the $\varepsilon^{\prime}$-phase or from the twin. On the streaks, however, the intensity maxima are observed the positions of which just coincide with the spots of the $\varepsilon^{\prime}$-phase and

*** In the calculations, the following values were used : $a=3.574$ $\AA$ for the matrix and $a=2.522 \AA, c=4.066 \AA$, and $c / a=1.612$ for $\varepsilon$-phase. These were estimated from the data given in (8) and (9).

(8) A. R. Troiano and F. T. Meguire: Trans. ASM, 31(1943), 340 .

(9) J. G. Parr: J. Iron and Steel Inst., 171 (1952), 137.

*** $(10 \overline{1} 2) \varepsilon$ and $(0 \overline{2} \overline{2}) T$ spots are found near $(020)$ spot, and $(\overline{1} 01 \overline{2})$ and $(022)_{T}$ spots are found near $(020)$ spot, and $(\overline{101} \overline{2})_{c}$ and $(022)_{T}$ spots are found near (020) spot, although these kinds of spots, do not lie on the same reciprocal plane, in the normal state. This is due to the fact that (101) plane is inclined clockwise about [101] axis. Therefore the inclination of the specimen from (101) plane is estimated to be about $10^{\circ}$, if it is assumed to be between the inclinations at which $(020)$ and $(0 \overline{2} \overline{2})_{T}$ appear, respectively. $(\overline{2} 02)$ and $(20 \overline{2})$ spots are not found, although they belong to the [101] zone. The reason will be that the (101) plane is inclined by $8^{\circ}$ clockwise about $[0 \overline{0} 0]$ axis. The spots from the $\varepsilon$-phase and the twins are not found, because their expected positions are the same as those of $(\overline{202})$ and $(20 \overline{2})$ spots. In the neighbourhood of (111) and (11i) spots, no spots can be seen at the positions corresponding to $(0 \overline{1} 1 \overline{2}) \varepsilon$ and $(01 \overline{1} 2) \varepsilon$ appearing near $(1 \overline{11})$ and (i11) spots. This is probably due to the bending of the specimen about the [111] axis, which can be recognized from extinction contours in (a). twins. Accordingly it is confirmed that both the $\varepsilon^{\prime}$-phase and the twins exist in the cold-rolled specimen. These streaks are also probably due to the fact that they are very thin or contain many faults.

To confirm that the spots which were interpreted as

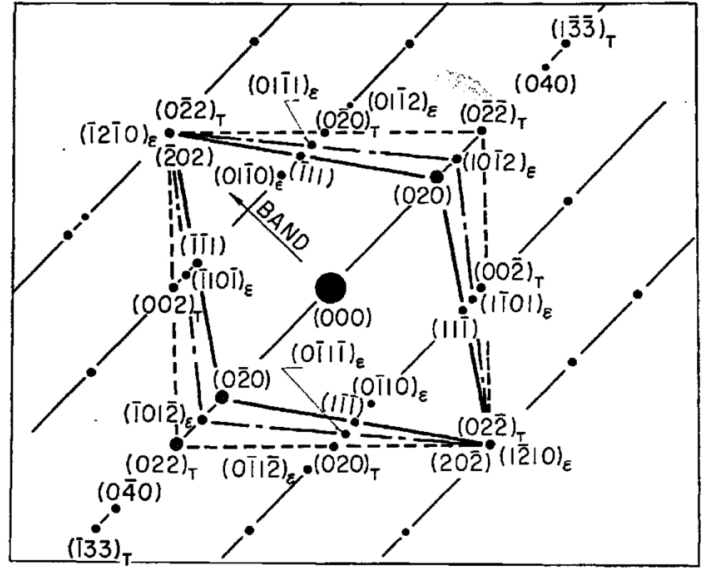

Fig. 2 Key diagram of Photo. $2(\mathrm{~b})$. Suffix ' $T$ ' means the the twin spots. Suffix ' $\varepsilon^{\prime}$ means the $\varepsilon^{\prime}$-phase spots. $[101]$ zone

- - - $[100]_{T \text { zone }}$
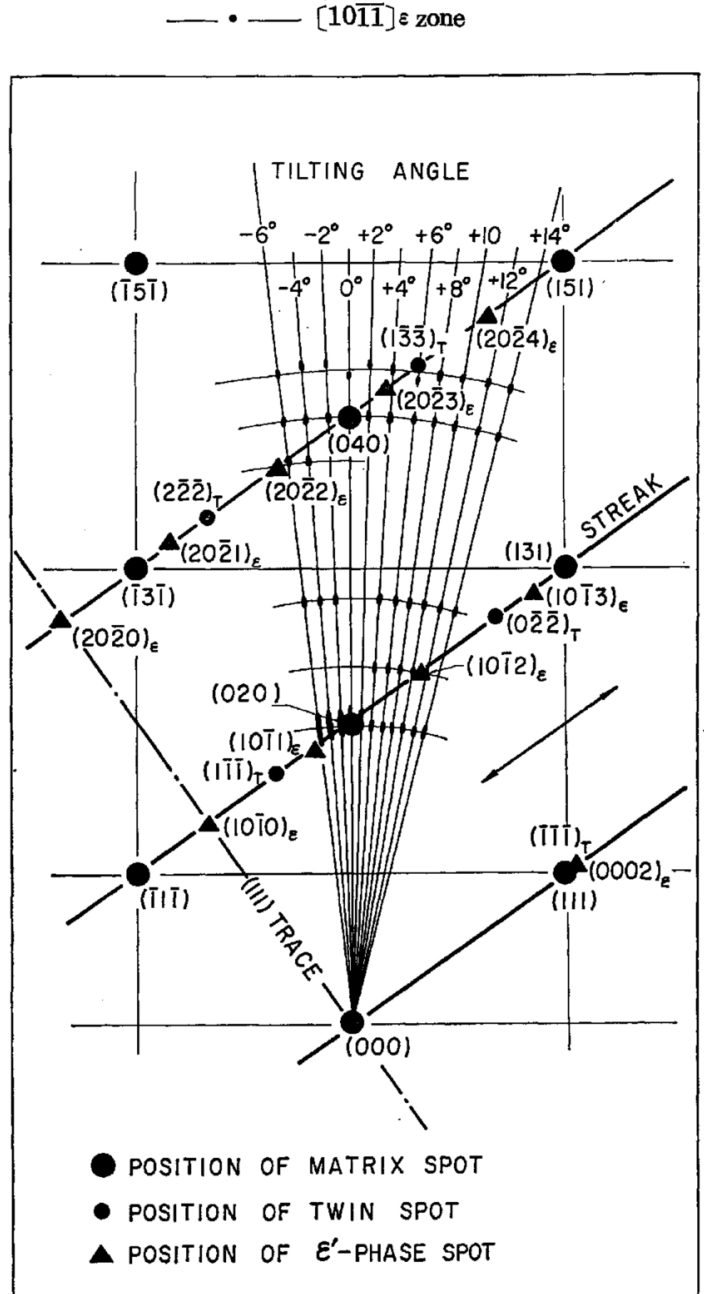

Fig. 3 Plot of reflection spots into a reciprocal lattice of the $[\mathbf{1 0} \overline{1}]$ zone, the specimen beine tilted. The arrow shows the direction of streaks.

those from the $\varepsilon^{\prime}$-phase and the twins are not mere intersections of the streks with Ewald sphere, an examination was made of the change of the diffraction pattern by 
tilting the specimen within the range of $20^{\circ}$ about the direction parallel to the bands in Photo. 2(b). In Fig. 3, the reciprocal lattice belonging to the $[10 \overline{1}]$ zone of the matrix is plotted superposing the $\varepsilon^{\prime}$ and twin spots which are predicted from the result of a trace analysis of bands that the $\varepsilon^{\prime}$-phase and the twins are formed on the (111) plane of the matrix. In this figure are also plotted the spots (e.g., those on OP line in Photo. 2(b)) observed in the patterns which were taken at intervals of $2^{\circ}$ within the tilting angles from $-6^{\circ}$ to $14^{\circ}$, the tilting axis being in the $[10 \overline{1}]$ direction. Let us first examine the spots due to the $\varepsilon^{\prime}$-phase. The $(20 \overline{2} 2)_{\varepsilon}$ spot is seen at the tilting angle of $-4^{\circ}$ and $-6^{\circ}$. At these angles there is a spot which seems to be due to the (10I1) reflection though it looks like an elongated (020) spot. The $(10 \overline{1} 2)_{\varepsilon}$ spot appears in the range from $+4^{\circ}$ to $+10^{\circ}$. If the plotted spots were due to the intersections of the streaks of the $\varepsilon^{\prime}$ or any other phase with the Ewald sphere, they would appear at different distances from the origin. But actually the spots appear on each circle with a definite radius corresponding to the normal $(10 \overline{1} 2)_{\varepsilon}$ spot or $(20 \overline{2} 2)_{\varepsilon}$ spot. Therefore these spots are considered not to be due to the intersections of the streaks but due to the normal spots of the $\varepsilon^{\prime}$-phase. $(20 \overline{2} 3)_{\varepsilon}$ and $(20 \overline{2} 4)_{\varepsilon}$ spots were not observed probably because of the small intensity due to the large Bragg angle.

Next let us examine the twin spots. The $(1 \overline{3} \overline{3})_{T}$ spot appears in the whole range of the tilting angle, and the $(0 \overline{2} \overline{2})_{T}$ spot also appears except at $0^{\circ}$ and $+2^{\circ}$. Each of them is always located at a constant distance from the origin for the varying tilting angles. Therefore these spots are considered not to be the intersections of the streaks but to be the normal twin spots as in the case of the $\varepsilon^{\prime}$-phase. From the above result, it is concluded that both the $\varepsilon^{\prime}$-phase and the twins are present in Photo. 2 (a). The $(020),(040),(1 \overline{3} \overline{3})_{T}$ and $(10 \overline{1} 2)_{\varepsilon}$ spots appear at every tilting angle. This resulted from the local rotation of the crystal due to the severe working of the material and the bending of the specimen during the thinning.

\section{Discussion}

As already described in the introduction, it has so far been discussed that the pronounced work-hardening of high manganese steel is caused by the occurrence of the $\alpha^{\prime}$-phase, $\varepsilon^{\prime}$-phase and stacking faults and solid solution hardening.

About the occurrence of the $\alpha^{\prime}$-phase, Colette et al. found it, in a Hadfield steel deformed by tension, using X-ray diffraction. Roberts ${ }^{(7)}$ first thought to have observed the $\alpha^{\prime}$-phase in a similar sample by means of electron microscopy, but afterwards ${ }^{(10)}$ he admitted that this conclusion resulted from a false interpretation of the electron diffraction patterns. The $\alpha^{\prime}$-phase was not detected in the present experiment. On the other hand Saito ${ }^{(11)}$, as pointed out by Buhr et al., (1) reported that a small amount of some ferromagnetic substance was found to exist on the slip planes in the hammered Hadfield steel by the magnetic colloid test and inferred that the substance was very thin $\alpha^{\prime}$-martensites. Thus it remains unsolved whether

(10) W. N. Roberts : Trans. AIME, 230 (1964), 372.

(11) T. Saito : J. Japan Inst. Metals, $27(1963), 376,467$. the $\alpha^{\prime}$-phase is produced in the deformed Hadfield steels.

Most of the recent studies asserted the existence of the $\varepsilon^{\prime}$-phase and the stacking faults in the deformed Hadfield steel and considered it as an origin of the work-hardening. Their existence may also be convinced from the fact that the stacking fault energy is small for high manganese steel. Since the $\varepsilon^{\prime}$-phase is given when the stacking of (111) planes in a f.c.c. crystal is faulted on every one layers, it is considered that the $\varepsilon^{\prime}$-phase and the stacking faults are closely related to each other and thus the probability of the occurrence of the two is high. The reason is therefore understood why they were found in the previous experiments ${ }^{(5)}$.

In the case in which the $\varepsilon^{\prime}$-phase is very thin or contains many faults, it will contribute to the hardening, even if the normal crystal of the $\varepsilon^{\prime}$-phase is not substantially hardened.

From the arrangement of atoms, the existence of the $\varepsilon^{\prime}$-phase and the stacking faults suggests a possibility of the twin formation, as confirmed by the present study. The deformation twin may easily occur in the case of the high speed deformation, although it is generally believed difficult to occur in f.c.c. metals. In fact, the twin was observed even in nickel ${ }^{(12)}$, if the explosion pressure was applied for the deformation.

Before the publication of this paper, Roberts communicated $^{(10)}$ to the present authors that he also observed the deformation twins in the high manganese-steel. But the spots in his diffraction pattern which were interpreted as those of the twin might as well be explained as the $\varepsilon^{\prime}$-phase spots, if the streaks due to the reciprocal lattice rods (relrod) along the [0001] direction of the $\varepsilon^{\prime}$-phase were taken into consideration, because his specimen foil was oriented so that the reciprocal lattice rods might be cut at a large angle by the Ewald sphere. In this respect, the extra spots which are observed between the matrix spots in Photo. 1 (b) can be interpreted as those of the twins, clearly distinguished from the intersections of the relrods of the $\varepsilon^{\prime}$-phase, because the rods almost lie on the Ewald sphere. In Photo.l the very thin deformation twins are clearly observed, but when the twins and the $\varepsilon^{\prime}$-phase plates coexist as shown in Photo. 2 , the distinction between them becomes too difficult to determine their thickness. It should therefore be noted that the value of the hardness, $500 \mathrm{VHN}$, is an average of the hardness of the regions where either the twin or the $\varepsilon^{\prime}$-phase was present and that of the regions where they coexisted.

The influences of different kinds of phases and faults on the hardness depend on their density and shape, though difficult to distinguish them. Roberts reported that when the spacing of the twins in high manganese steel was $2000 \AA$ and $500 \AA$, the hardness was 400 VHN and 600 VHN, respectively. Therefore, the hardness seems to show a considerable change with the spacing of the twins. The reason why the Hadfield steel shows the most remarkable work hardening among many kinds of manganese steel, may be attributed to the small stacking fault energy in this composition which is liable to produce the $\varepsilon^{\prime}$-phase, stacking faults and the twins.

(12) R. L. Nolder and G. Thomas : Acta Met., 11 (1963), 994. 


\section{Conclusion}

It was previously reported that the $\varepsilon^{\prime}$-phase and stacking faults were produced in a hammered high manganese steel. The present experiment confirmed the existence of deformation twins together with them in the Hadfield steel cold-rolled by $30 \%$ reduction. The deformation twins will contribute to the pronounced work-hardening of high manganese steel as well.

The authors wish to thank Mr. A. Kore'eda for taking electron micrographs. 